\title{
Increasing Broadcast Reliability in Vehicular Ad Hoc Networks
}

\author{
Nathan Balon and Jinhua Guo \\ Dept. of Computer and Information Science, University of Michigan, Dearborn \\ Dearborn, MI, USA \\ \{nbalon,jinhua\}@umd.umich.edu
}

\begin{abstract}
Broadcast transmissions are the predominate form of network traffic in a VANET. However, since there is no MAC-layer recovery on broadcast frames within an 802.11-based VANET, the reception rates of broadcast messages can become very low, especially under saturated conditions. In this paper, we present an adaptive broadcast protocol that improves the reception rates of broadcast messages. We rely on the observation that a node in a VANET is able to detect network congestion by simply analyzing the sequence numbers of packets it has recently received. Based on the percentage of packets that are successfully received in the last few seconds, a node is able to dynamically adjust the contention window size and thus improve performance.
\end{abstract}

\section{Categories and Subject Descriptors}

C.2.2 [Network Protocols]: Protocol architecture

\section{General Terms}

Design

\section{Keywords}

VANET, DSRC, IEEE 802.11, broadcast, contention window, prioritized access

\section{INTRODUCTION}

Dedicated Short-Range Communications [1] (DSRC) offers the potential to effectively support vehicle-to-vehicle and vehicle-toroadside safety communications. A larger portion of the messages sent on the control channel of DSRC are broadcast. Some uses for broadcasts are sending emergency warnings and periodically broadcasting a vehicle's state. The lower layers of DSRC are a variant of IEEE 802.11a [2]. However, 802.11 is known for not being able to manage the medium very efficiently, especially in case of broadcast messages. Providing reliable delivery of broadcast messages in a VANET introduces several key technical challenges.

No retransmission is possible for failed broadcast transmissions. A failed unicast transmission is detected by the lack of an acknowledgment (ACK) from the receiver. However, acknowledgments are not used for broadcast messages.

The contention window $(\mathrm{CW})$ size fails to change because of the lack of MAC-level recovery. As a result, the $\mathrm{CW}$ is held constant for broadcast transmissions.

Copyright is held by the author/owner(s).

VANET'06, September 29, 2006, Los Angeles, California, USA

ACM 1-59593-540-1/06/0009.
The hidden terminal problem exists because of the lack of the RTS/CTS exchange.

The vehicular network must support the ability to prioritize messages. When an emergency warning is broadcast, it should be given a high priority.

The goal of this paper is to develop an adaptive broadcast protocol that improves the reliability of delivering broadcast messages in a VANET.

\section{RELATED WORK}

A number of authors have addressed the problem of sending broadcast messages in a VANET. Torrent-Moreno et al. [3] show that the probability of reception of a broadcast message decreases as the distance from the sender increases and under saturated conditions the probability of reception of a message can become very low. Xu et al. [5] propose a single-hop broadcast protocol that increases the probability of a message's reception by sending the message multiple times. Yang et al. [6] propose the VCWC protocol to transmit emergency warning messages (EWM), which is based on a state machine and a multiplicative rate decrease algorithm. Both [5] and [6] aim at increasing the probability of reception by broadcasting a message multiple times.

\section{ADAPTIVE CONTENTION WINDOW ADJUSTMENT}

According to the DSRC standard each vehicle broadcasts its status to its neighbors approximately 10 times every second [4]. As such, a node in a VANET is able to detect collisions and congestion by analyzing the sequence numbers of the packets it has recently received. While a node does not know if the packets it sent are correctly delivered, it can estimate the percentage of packets sent to it from its neighboring nodes that are successfully received. Based on the observation of packets that are recently received, a node is able to determine the current local conditions of the network. Therefore, a node is able to dynamically adjust its transmission parameters.

The sequence control field of an 802.11 MAC header contains a 12-bit sequence number. As a result, each node records the overheard sequence numbers. As shown in Fig. 1, node $A$ records that it has overheard the frames coming from node $B$ with the sequence numbers $32,34,35,36,37,38,40,41$. Based on the observed sequence numbers node $A$ can conclude that frame 33 and frame 39 were corrupted or lost and that $20 \%$ of the frames sent from node $B$ were not received by node $A$.

To determine the local state of the network, a node maintains a table with entries for neighboring nodes from which it has recently received frames. The table entries contain: a MAC address, the last sequence number, a weighted reception rate, and a timestamp. 


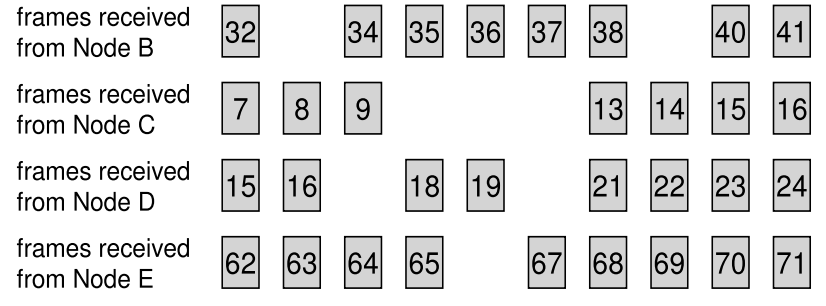

Figure 1: Received Frames at Node $A$

To begin, the last sequence number is used to detect gaps between the sequence numbers. Next, the weighted reception rate is used to determine the percentage of packets that are successfully received from a specific node. Last, the timestamp is used to remove entries from the table if a message has not been heard from that node within a specified time period.

A weighted reception rate is used to put more emphasis on recent events. Equation 1 is used to calculate the weighted reception rate. Each time a frame is successfully received, the weighted reception rate is recalculated. The variable $\alpha$ is used to put more or less weight on the current network condition.

$$
\text { EstRecp }=\alpha * \text { EstRecp }+(1-\alpha) * \text { SampledRecp }
$$

The nodes also maintain a timer. When the timer expires, the local reception rate is determined and the $\mathrm{CW}$ is adjusted. The local reception rate is calculated using Equation 2, which is the average of the weighted reception rates.

$$
\text { LocalRecpRate }=\Sigma \text { EstRecp } / \text { Nodes }
$$

After the local reception rate is calculated, it is compared against the previously stored local reception. The node also maintains a threshold value that it uses to determine whether to increase or decrease the $\mathrm{CW}$. If the new local reception rate decreases by a value greater than the threshold, the $\mathrm{CW}$ is increased. On the other hand, if new local reception rate increases by a value greater than the threshold, the $\mathrm{CW}$ is decreased. As a result, the $\mathrm{CW}$ adapts to the condition of the network. The node then uses the new CW for all broadcast transmission until the next time the local reception rate is sampled.

In order to prioritize access $802.11 \mathrm{e}$ is used. Access priority is achieved through different choices of inter-frame spacing (IFS) and CW sizes. High priority classes in turn use a shorter IFS and a smaller CW.

\section{PERFORMANCE EVALUATION}

The ns-2 network simulator was used to evaluate the proposed broadcast algorithm. For the simulations, an eight-lane stretch of freeway models the vehicular environment.

The simulations contain the three traffic classes displayed in Table 1 . The classes $T C[0]$ and $T C[1]$ account each for approximately $5 \%$ of the total network traffic. These two classes represent event-driven traffic. In comparision, $T C[2]$ is used for vehicles to exchange their state every $100 \mathrm{~ms}$ and represents approximately $90 \%$ of the network traffic.

In order to determine how well the proposed algorithm performs under various conditions, eight different scenarios were generated. Each successive scenario increased the number of vehicles in the network by 50 vehicles. Furthermore, two set of simulations were conducted. The first set of simulations compared the performance of the $802.11 \mathrm{e}$ protocol under various loads. The second set of
Table 1: Traffic Classes

\begin{tabular}{|l|l|l|l|}
\hline Traffic Class & $A I F S$ & $C W_{\min }$ & $C W_{\max }$ \\
\hline 0 & 2 & 7 & 15 \\
1 & 2 & 15 & 63 \\
2 & 3 & 31 & 1023 \\
\hline
\end{tabular}
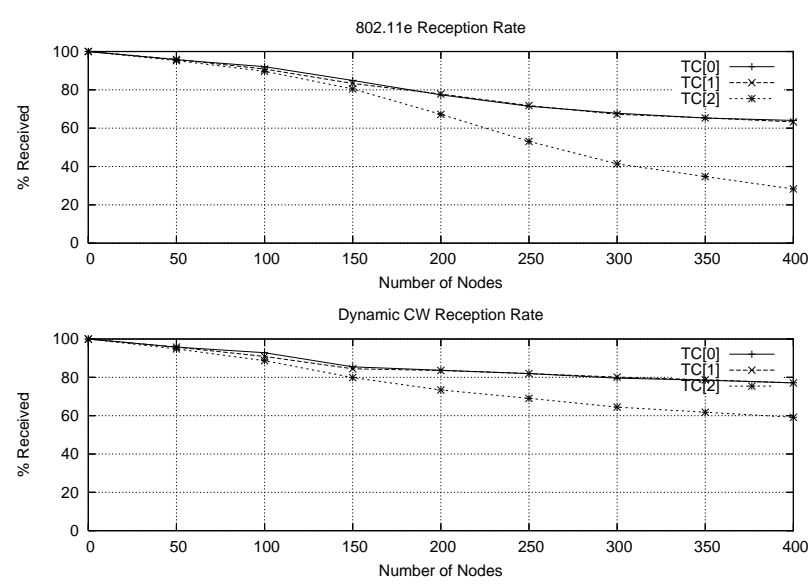

Figure 2: Reception Rate

simulations examined the effect that varying the size of the CW has on the probability of a message's reception. Figure 2 contains the results of the simulations. As a result of adaptively adjusting the CW size, the probability of a collision is reduced.

\section{CONCLUSION}

Adaptively adjusting the contention window size increases the probability of reception for broadcast traffic. The presented solution consumes no additional network resource and little additional complexity for the mobile nodes. Our future work will focus on adaptive control of the transmission power, based on the observed network conditions.

\section{ACKNOWLEDGMENTS}

This research work was supported in part by the US NSF Grant CNS-0521142 and University of Michigan, Office of Vice President for Research (OVPR) Grant.

\section{REFERENCES}

[1] L. Armstrong. Dedicated short-range communications project.

[2] Federal Communications Commission. Amendment of the commission's rules regarding dedicated short-range communication service in the 5.850-5.925 ghz band, FCC 02-302. Technical report, FCC, November 2002.

[3] M. Torrent-Moreno, D. Jiang, and H. Hartenstein. Broadcast reception rates and effects of priority access in 802.11-based vehicular ad-hoc networks. In Proceedings of the First International Workshop on Vehicular Ad Hoc Networks, 2004, Philadelphia, PA, USA, October 1, 2004, pages 10-18. ACM, 2004.

[4] Vehicle Safety Communications Consortium. Vehicle safety communications project task 3 final report: Identify intellegent vehicle safety applications enabled by DSRC, March 2005.

[5] Q. Xu, R. Sengupta, and D. Jiang. Design and analysis of highway safety communication protocol in $5.9 \mathrm{ghz}$ dedicated short range communication spectrum, Spring 2003.

[6] X. Yang, J. Liu, F. Zhao, and N. H. Vaidya. A vehicle-to-vehicle communication protocol for cooperative collision warning. In MobiQuitous, pages 114-123. IEEE Computer Society, 2004. 